\title{
Implantable rectenna system for biomedical wireless applications
}

\author{
Shuoliang Ding \\ Group of Electrical Engineering -Paris, \\ UMR 8507 CNRS, \\ CNRS, CentraleSupelec, Université \\ Paris-Sud, Sorbonne Université \\ Gif-sur-Yvette, France \\ shuoliang.ding@geeps.centralesupelec.
}

\author{
Stavros Koulouridis \\ Electrical and Computer Engineering \\ Department \\ University of Patras \\ Patras Greece \\ Koulouridis@upatras.gr
}

\author{
Lionel Pichon \\ Group of Electrical Engineering -Paris, \\ UMR 8507 CNRS, \\ CNRS, CentraleSupelec, Université \\ Paris-Sud, Sorbonne Université \\ Gif-sur-Yvette, France \\ lionel.pichon@geeps.centralesupelec.fr
}

\begin{abstract}
In this paper, a complete RF to DC wireless power transmission implantable rectenna system is presented. For simplicity, an external half-wave dipole at Industrial, Scientific and Medical bands (ISM 902.8-928 MHz) is selected as an energy emitter from outside human body. An embedded circular dipole antenna receives the energy and then converts it to DC power by a rectifying circuit. The structure of the system is discussed in details. Finally, the rectifying efficiency and the global system's efficiency are examined for different external antenna to human body distances, different embedded depth and various levels of circuit's input power.
\end{abstract}

Keywords-Wireless power transmission system, Rectenna, Rectifying efficiency, Industrial, Scientific and Medical (ISM) bands

\section{INTRODUCTION}

Wireless energy transmission has gained significantly attention in biomedical domain during those recent years. An implantable medical devices (IMD) could have various type of uses like condition monitoring, drug delivery, information exchange, etc. [1] In comparison with near-field inductive charging, far-field radiative charging can be used with a higher implantable depth and is much more robust to the change of antenna locations, directions and surrounding environment.

A Radio Frequency (RF) medical energy transmission system normally consists of 2 parts: An antenna for capturing energy and a circuit for converting alternate current (AC) power to direct current (DC) power. There are many scientific publications on the antenna design [2][3]. Moreover, the rectenna system has also been studied by many research teams in the world [4]: H. W. Cheng et. al. has studied in [5] a rectenna system that operates at $400 \mathrm{MHz}$ with a circuit input power of $10 \mathrm{dBm}$ and rectifying efficiency of $76 \%$; B. J. DeLong et. al. has proposed a rectenna structure that operates at $2.4 \mathrm{GHz}$ in free space, but the efficiency at $-20 \mathrm{dBm}$ is only around $7 \%$ [6]; C. Liu et. al. has worked on an rectenna system which is implanted in body but only have an efficiency of $20 \%$ with an input power of $-20 \mathrm{dBm}$ and implantation depth of $4 \mathrm{~mm}$ [7]. In conclusion, due to its high operating frequencies and low input power level, the design of a deep-implanted and efficient energy transmitting rectenna is still a challenge for researchers.

In this paper, a deep-implanted rectenna system with a low power input below $-20 \mathrm{dBm}$ is examined. The power transmission structure is presented, important characteristics of the embedded antenna are detailed and a global co-simulation involving both the antenna part and the rectifying circuit is performed. The system is analyzed in a 3D realistic simulation environment: the entire rectenna system is embedded in a three-layer (skin, muscle et bone) cylindrical human arm model. Numerical results are also presented.

\section{RECTIFYING SYSTEM DESIGN}

This section presents the structure of the entire power rectifying system.

A. Power conversion system structure

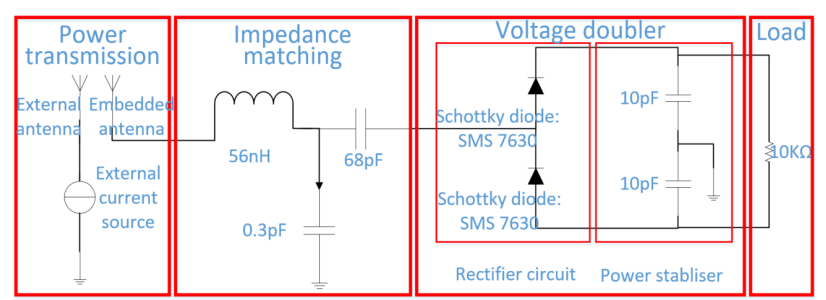

Fig.1. Rectenna system structure

Figure 1 shows a detailed design of the rectifying system including all the components and their values. This system has four sections in total. The first one is the power transmission part proposed in [8], an external dipole transmits microwave power to a circular dual-band dipole antenna which is embedded in-body and resonates at $402 \mathrm{MHz}$ and $915 \mathrm{MHz}$. The dipole antenna operates at the power transmission frequency band: $902.8-928 \mathrm{MHz}$. The 
second section is the impedance matching circuit, minimizing the mismatch loss and reducing the reflection. The third section is a standard voltage doubler using the SMS 7630 Schottky diode fabricated by the Skyworks company and two capacitors of $10 \mathrm{pF}$ as voltage stabilizer. The final section is a $10 \mathrm{~K} \Omega$ load that simulates the energy consuming part in real case.

\section{B. Power Transmission model}

Power transmission scenario is shown in figure 2. A three-layer cylindrical human arm model (radius are: Bone $0-25 \mathrm{~mm}$; Muscle 25-47.5mm; Skin 47.5-50mm) is used as simulation environment. In order to save the calculation time, the length of the arm model is set to the minimum value such as does not affect the results. An external half wavelength dipole radiates energy from outside the arm to the deep-implanted in muscle (more than $10 \mathrm{~mm}$ from skin) circular dipole. S21 and other necessary power transmitting values are all presented in [8]. This system operates at $915 \mathrm{MHz}$ (ISM / Industrial, Scientific, and Medical band). As discussed in [8], the maximum power received by the embedded antenna (the circular dipole) when the external antenna is over $200 \mathrm{~mm}$ away from body is lower than $20 \mathrm{dBm}$ since the maximum authorized radiated power by the external antenna at this frequency band is $30 \mathrm{dBm}$ [9]. Also in this case, the Special Absorption Rate (SAR) values are $0.28 \mathrm{~W} / \mathrm{kg}$ and $0.19 \mathrm{~W} / \mathrm{kg}$ for every $1 \mathrm{~g}$ average and 10 $\mathrm{g}$ average of the human tissue, which satisfy the 2 IEEE standards about SAR limits [10] [11], and ensures the safety level of the embedded antenna (circular dipole).

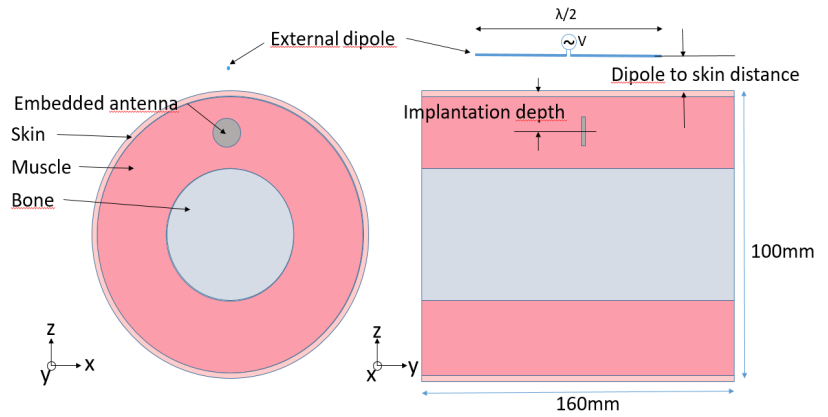

Fig. 2. Power transmission link design

\section{Power Transmitting Antenna Design}

The embedded antenna is a circular antenna. The detailed design is shown in figure 3 .

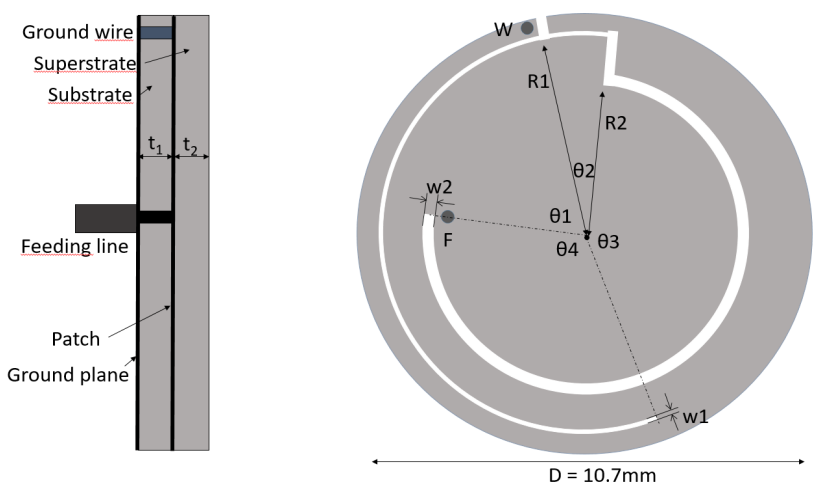

Fig. 3. Detailed design of the reception antenna

The antenna is made up of a radiating slot patch with a ground plane. The patch and the ground plane are grounded by a ground wire of radius $=0.15 \mathrm{~mm}$ that is located at point W (see Fig.3). Two circular slots are cut away from the patch. The patch is printed on a layer of substrate made of Rogers RO $3210\left(\varepsilon_{\mathrm{r}}=10.2, \tan \delta=0.003\right)$ which has a thickness of $t_{1}=0.64 \mathrm{~mm}$. The antenna is fed by a coaxial cable at point $\mathrm{F}$ and the patch is covered by a circular superstrate that of the same material and size as the substrate. All detailed parameters are presented in Table. I.

TABLE I. ANTENNA PARAMETERS

\begin{tabular}{cccc}
$\begin{array}{c}\text { Parameter } \\
\text { Name }\end{array}$ & Value (mm) & $\begin{array}{c}\text { Parameter } \\
\text { Name }\end{array}$ & Value (deg) \\
\hline R1 & 4.9 & $\theta 1$ & 70 \\
R2 & 3.76 & $\theta 2$ & 18 \\
w1 & 0.15 & $\theta 3$ & 163 \\
w2 & 0.32 & $\theta 4$ & 109 \\
t1 & 0.64 & & \\
t2 & 0.64 & & \\
D & 10.7 & & \\
\hline
\end{tabular}

The reflection coefficient of the antenna at $915 \mathrm{MHz}$ and for different implantation depths is shown in figure 4. As seen, it is practically unaffected by the related position inside the arm model.

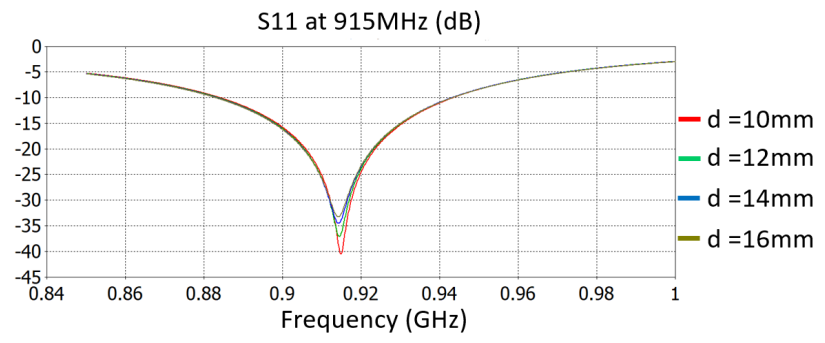

Fig. 4. S11 of the circular dipole antenna at $915 \mathrm{MHz}$ band

\section{Rectifying Circuit Design}

Once the power transmission model is established, the choice of the non-linear component for the rectifying circuit is of vital importance. The chosen diode must be adapted to high frequency and have low power consumption since the input is low. As mentioned in [12], the Schottky diode SMS 7630 fabricated by Skyworks company has good low-power performance and thus is chosen to be used with an input below $-20 \mathrm{dBm}$. 
Furthermore, an impedance matching circuit is optimized between the embedded antenna (circular dipole) and the rest of circuit in order to reduce the reflection loss and the power consumption of the components.

\section{E. Numerical Results}

The global simulation is performed by CST [13] that supports at the same time electromagnetic modeling and circuit simulations. All the circuits are off-the-shelf components and are based on spice models. The rectifying efficiency is calculated by the ratio between the power consumed by the load and the power received by the embedded antenna (circular dipole). Global system efficiency is defined as the ratio between power consumed by the load and the power emitted by the external dipole. Table II shows the relationship between the global system efficiency, rectifying efficiency, the distance between the two antennas and the embedded depth of the circular dipole.

\section{TABLE II. RECTIFYING EFFICIENCY OF THE CIRCUIT}

\begin{tabular}{ccccc}
$\begin{array}{c}\text { Embedde } \\
\text { d depth } \\
\text { (mm) }\end{array}$ & $\begin{array}{c}\text { Dipole } \\
\text { to skin } \\
\text { distance } \\
\text { (mm) }\end{array}$ & $\begin{array}{c}\text { Power } \\
\text { consumed } \\
\text { by load } \\
\text { (dBm) }\end{array}$ & $\begin{array}{c}\text { Rectifying } \\
\text { efficiency } \\
\mathbf{( \% )}\end{array}$ & $\begin{array}{c}\text { Global } \\
\text { system } \\
\text { efficiency } \\
\text { (\%) }\end{array}$ \\
\hline $\mathbf{1 0}$ & $\mathbf{1 7 5}$ & -26.71 & 24.00 & 0.213 \\
$\mathbf{1 0}$ & $\mathbf{2 5 0}$ & -30.31 & 17.74 & 0.093 \\
\hline $\mathbf{1 0}$ & $\mathbf{5 5 0}$ & -42.04 & 6.37 & 0.006 \\
\hline $\mathbf{1 6}$ & $\mathbf{1 7 5}$ & -30.35 & 18.47 & 0.092 \\
$\mathbf{1 6}$ & $\mathbf{2 5 0}$ & -35.01 & 11.91 & 0.032 \\
$\mathbf{1 6}$ & $\mathbf{5 5 0}$ & -48.01 & 2.76 & 0.001 \\
\hline
\end{tabular}

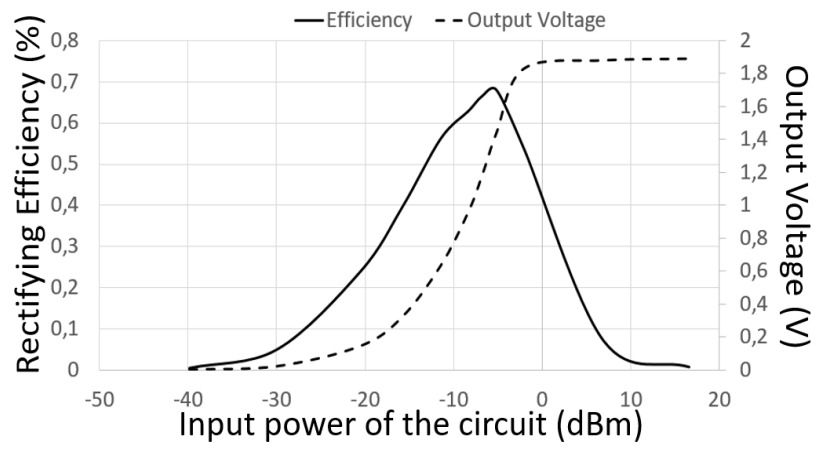

Fig.5. Efficiency and output voltage results for the rectifying circuit

From the results shown in Table II, when the dipole emits $30 \mathrm{dBm}$ power, the rectifying efficiency of the circuit can reach $24 \%$ when the received power by the embedded antenna (circular dipole) is $-20 \mathrm{dBm}$ and load is $10 \mathrm{~K} \Omega$. However, the efficiency is also relatively low when the inbody antenna is implanted deeper in the body or the external antenna is far from the body with all the loss at each component. Figure 5 shows the circuit's rectifying efficiency as a function of the circuit's input power. With the existence of the non-linear component, there is a phase delay between the current and the voltage. Thus, input power is calculated by:

$$
P_{\text {active }}=U_{r m s} * I_{r m s} * \cos \varphi_{c}
$$

where $U_{\text {rms }}$ and $I_{\text {rms }}$ are the root mean square (rms) values of the voltage and the current right after the receiver antenna (circular dipole) respectively and $\varphi_{c}$ is their phase difference. According to Figure 3, the efficiency of the rectifying circuit can reach up to $67.9 \%$ when the input power of the circuit is $-5.1 \mathrm{dBm}$.

\section{CONCLUSION}

A wireless RF to DC implantable power rectifying system for biomedical applications is presented in this paper. System operates at $915 \mathrm{MHz}$ ISM band. With the limitation of radiated power $(30 \mathrm{dBm})$, a series of numerical experiments is carried out in order to calculate efficiency for various implantation depths of the embedded antenna (circular dipole) and various distances between the external dipole and the human arm. For embedded depth of $10 \mathrm{~mm}$ and skin to external dipole distance equal to $175 \mathrm{~mm}$, the input power of the circuit is estimated to $-20 \mathrm{dBm}$ and a rectifying efficiency of $24 \%$ is obtained for a $10 \mathrm{~K} \Omega$ load. Moreover, for the above scenario, the DC power rectified by this system is capable of feeding simultaneously two sensors that mentioned in [14] which requires only $1.1 \mu \mathrm{W}$ each. It is noted that the rectifying efficiency can theoretically reach $67.9 \%$ for the presented circuit as shown in Figure 5.

In future work, the rectifier circuit will be fabricated and experimentally validated. Some measurement results will be presented at the conference.

\section{REFERENCES}

[1] K.S.Nikita. (2010). Handbook of Biomedical Telemetry, Wiley, 2013, pp. 14

[2] S. Bakogianni, and S. Koulouridis, "An implantable planar dipole antenna for wireless medradio-band biotelemetry devices," IEEE Antennas Wireless Propag. Lett., vol. 15, pp. 234-237, 2016.

[3] Kiourti, A., \& Nikita, K. S. (2012). "Miniature scalp-implantable antennas for telemetry in the MICS and ISM bands: Design, safety considerations and link budget analysis." IEEE Transactions on Antennas and Propagation, 60(8), 3568-3575.

[4] Essakhi, B., Akoun, G., \& Pichon, L. (2007). A global time domain circuit simulation of a microwave rectenna, International Journal of Numerical Modelling: Electronic Networks, Device and Fields (November 2006), 3-15.

[5] H. Cheng, T. Yu and C. Luo, "Direct current driving impedance matching method for rectenna using medical implant communication service band for wireless battery charging," in IET Microwaves, Antennas \& Propagation, vol. 7, no. 4, pp. 277-282, 19 March 2013.

[6] B. J. DeLong, A. Kiourti and J. L. Volakis, "A Radiating Near-Field Patch Rectenna for Wireless Power Transfer to Medical Implants at $2.4 \mathrm{GHz}, "$ in IEEE Journal of Electromagnetics, $R F$ and Microwaves in Medicine and Biology, vol. 2, no. 1, pp. 64-69, March 2018.

[7] C. Liu, Y. Guo, H. Sun and S. Xiao, "Design and Safety Considerations of an Implantable Rectenna for Far-Field Wireless Power Transfer," in IEEE Transactions on Antennas and Propagation, vol. 62, no. 11, pp. 5798-5806, Nov. 2014.

[8] Ding, S., Koulouridis, S., \& Pichon, L. (n.d.). "A Dual-Band Miniaturized Circular Antenna for Deep in Body Biomedical 
Wireless Applications," 2019 European Conference on Antenna and Propagation, EUCAP, Poland, 2019, pp 1-5.

[9] FCC 15. 209, Standard Specification for Radiated emission limits, general requirements.

[10] IEEE standard for safety levels with respect to human exposure to radiofrequency electromagnetic fields, $3 \mathrm{kHz}$ to $300 \mathrm{GHz}$, IEEE Standard C95.1, 1999.

[11] IEEE standard for safety levels with respect to human exposure to radiofrequency electromagnetic fields, $3 \mathrm{kHz}$ to $300 \mathrm{GHz}$, IEEE Standard C95.1, 2005

[12] M. ur Rehman, W. Ahmad and W. T. Khan, "Highly efficient dual band 2.45/5.85 GHz rectifier for RF energy harvesting applications in ISM band," 2017 IEEE Asia Pacific Microwave Conference (APMC), Kuala Lumpar, 2017, pp. 150-153.

[13] Computer Simulation Technology (CST) STUDIO SUITE. Ver 2017, CST AG, Germany

[14] Wu, T., Law, M., Mak, P., Member, S., \& Martins, R. P. (2013). “An Ultra - Low Power CMOS Smart Temperature Sensor for Clinical Temperature Monitoring." 2013 IEEE International Conference of Electron Devices and Solid-State Circuits, (3), 1-2. 